\title{
BMJ Open Effects of living kidney donation on arterial stiffness: a systematic review protocol
}

\author{
Rosendo A Rodriguez (D) , ${ }^{1}$ Mayra Trentin Sonoda, ${ }^{2}$ Mohsen Agharazii, ${ }^{3}$ \\ Risa Shorr, ${ }^{4}$ Kevin D Burns ${ }^{2}$
}

To cite: Rodriguez RA, Trentin Sonoda M, Agharazii M, et al. Effects of living kidney donation on arterial stiffness: a systematic review protocol. BMJ Open 2021;11:e045518. doi:10.1136/ bmjopen-2020-045518

- Prepublication history and additional material for this paper is available online. To view these files, please visit the journal online (http://dx.doi.org/10. 1136/bmjopen-2020-045518).

Received 02 October 2020 Revised 06 February 2021 Accepted 17 February 2021

Check for updates

(C) Author(s) (or their employer(s)) 2021. Re-use permitted under CC BY-NC. No commercial re-use. See rights and permissions. Published by BMJ.

${ }^{1}$ Department of Medicine, The Ottawa Hospital, University of Ottawa, Ottawa, Ontario, Canada ${ }^{2}$ Division of Nephrology, Kidney Research Centre, Ottawa Hospital Research Institute, University of Ottawa, Ottawa, Ontario, Canada

${ }^{3}$ Division of Nephrology, CHU de Québec-Université Laval Research Centre, Quebec City, Québec, Canada

${ }^{4}$ Learning Services, The Ottawa Hospital, Ottawa, Ontario, Canada

Correspondence to Dr Rosendo A Rodriguez; rrodriguez1847@gmail.com

\section{ABSTRACT}

Introduction Kidney donors have been reported to have accelerated progression of aortic stiffness and decreased glomerular filtration compared with healthy non-donors. This is a concern because increased aortic stiffness is an independent predictor of overall cardiovascular disease and all-cause mortality in the general population. To confirm if arterial stiffness increases after donation, we will systematically review all studies that evaluated indices of arterial stiffness in healthy individuals who underwent unilateral nephrectomy for kidney donation compared with age-matched healthy non-nephrectomised controls.

Methods/analysis We will comprehensively search for studies published between 1 January 1960 and 15 March 2021 in MEDLINE, EMBASE, Cochrane Central, OVID and EBM reviews. All prospective (cohort, case-control, case series and before-and-after studies) and retrospective non-randomised studies reporting indices of arterial stiffness in nephrectomised and non-nephrectomised healthy participants will be included. Primary outcome will be the difference in the functional metrics of arterial stiffness between donors and non-donors. Secondary outcomes will be the differences in systolic/diastolic blood pressures, serum creatinine, glomerular filtration, carotid artery intima-media thickness and vascular calcification. Study screening, selection and data extraction will be performed by two independent reviewers. Risk of bias will be independently assessed with the ROBINS-I tool and confidence in evidence by the Grading of Recommendations Assessment, Development and Evaluation recommendations. Qualitative and quantitative data syntheses as well as clinical and statistical heterogeneity (Forest plots, $I^{2}$ and Cochran's $Q$ statistics) will be evaluated. If clinical and statistical heterogeneity are acceptable, inverse variance-weighted effects will be analysed by random effect models.

Ethics and dissemination No ethical approval is necessary. Our results will be disseminated through peerreview publication and presentations to guide stakeholders on the evaluation and follow-up care of kidney donors. PROSPERO registration number CRD42020185551.

\section{INTRODUCTION}

Live donor kidney transplantation is the preferred treatment for patients with endstage renal disease (ESRD); it provides increased patient and graft survival and

\section{Strengths and limitations of this study}

This systematic review will synthesise best quality evidence on functional and structural components of arterial stiffness in kidney donors and age-matched healthy non-nephrectomised controls.

- Since systemic blood pressure may be a determinant of arterial stiffness, its inclusion in a secondary analysis will improve our understanding on the potential relationship between blood pressure and arterial stiffness after donation.

- Quality of evidence and differences in study design can limit the strength of our associations. To minimise misinterpretations, our study will implement well-validated scales for assessing risk of bias and confidence in cumulative evidence.

better quality of life than either dialysis or deceased donor transplantation. ${ }^{1}{ }^{2}$ Every year, nearly 20000 healthy adults worldwide accept the risk of donor nephrectomy to help family members, friends or even strangers to improve survival and quality of living. ${ }^{34}$ From a medical and ethical perspective, we ought to minimise their risks and maximise safety during and after kidney donation.

Although several studies ${ }^{25} 6$ have indicated that the absolute risk for healthy kidney donors following nephrectomy is very low, some have reported that unilateral nephrectomy is associated with an accelerated progression of aortic stiffness, enhanced left ventricular mass, interventricular septum thickness and decreased glomerular filtration compared with healthy non-donors matched by age and sex. ${ }^{7-11}$ In most of these reports, blood pressure at follow-up was not significantly different between donors and non-donors. ${ }^{891213}$ Moreover, the risk of developing hypertension after donation was associated with higher blood pressure readings before donation, suggesting that reduced glomerular filtration rate (GFR) postdonation may be a graded risk factor for increased 
aortic stiffness, independent of blood pressure. ${ }^{713}$ These findings are clinically relevant because increased aortic stiffness as measured by carotid-femoral pulse wave velocity (cf-PWV) is a strong independent predictor of overall cardiovascular disease and all-cause mortality in the general population. ${ }^{14} \mathrm{An}$ increase of $1 \mathrm{~m} / \mathrm{s}$ in cf-PWV in an otherwise healthy individual has been associated with an adjusted risk increase of $14 \%$ and $15 \%$ in total cardiovascular events and all-cause mortality. ${ }^{14}$ Although compensatory renal hypertrophy and glomerular hyperfiltration occur after unilateral nephrectomy, a net decrease in total GFR typically follows, and this raises the possibility that cardiovascular disease risk after donation may become comparable to the risk in patients with mild to moderate chronic kidney disease.$^{15-17}$ A reduction in renal function of such magnitude may be particularly critical for young living kidney donors who have the longest duration of risk exposure to the effects of reduced renal mass. To determine if kidney donors are at risk for increased aortic stiffness, a systematic appraisal of existing studies on arterial stiffness in donors compared with agematched healthy controls is necessary. This review differs from existing systematic reviews and meta-analyses that have evaluated the interventional effects of kidney transplantation on arterial stiffness in kidney recipients with ESRD. ${ }^{18} 19$ The results of this new review can highlight strengths and weaknesses in previous studies with kidney donors and determine if there is a need for further welldesigned investigations.

\section{Objectives}

In this study, we will systematically review all nonrandomised studies that evaluated central or peripheral arterial stiffness in healthy individuals who underwent unilateral nephrectomy after fulfilling standard criteria for kidney donation and who had measurements of the functional or structural components of arterial stiffness, as defined by validated metrics including: cf-PWV, brachial-ankle pulse wave velocity (PWV), pulse wave analysis, femoral-tibial PWV, ankle-brachial index, aortic distensibility, carotid artery intima-media thickness and vascular calcification. The major objective of this review will be to provide best quality evidence on whether arterial stiffness increases after living kidney donation beyond what is expected for age-matched healthy nonnephrectomised controls. We will primarily determine differences in functional markers of arterial stiffness between kidney donors and their respective comparative healthy non-donor controls. Since progression of arterial stiffness in kidney donors may be a determinant of cardiovascular outcomes, we will additionally compare arterial stiffness in donors before and after nephrectomy. Secondarily, we will document differences in systolic and diastolic blood pressure (SBP, DBP), serum creatinine, estimated GFR (eGFR) and structural markers of arterial stiffness (carotid intima-media thickness, arterial calcification scores) between these two populations.

\section{METHODS AND ANALYSIS}

We will conduct this review in accordance with the Cochrane Collaboration principles for Systematic Reviews $^{20}$ and this protocol has been registered in the PROSPERO register for systematic reviews. ${ }^{21}$ The preferred reporting items for systematic Reviews described by the Preferred Reporting Items for Systematic Reviews and Meta-Analyses guidelines ${ }^{22}$ will be followed and a checklist file for these recommendations will be included (online supplemental appendix 1). ${ }^{23}$

\section{Population and eligibility criteria \\ Kidney donors}

We will include healthy adults ( $\geq 18$ years old) of any age who underwent unilateral nephrectomy after meeting standard screening criteria for organ donation and whose arterial stiffness was measured non-invasively before and/ or after nephrectomy with any of the following functional metrics of arterial stiffness: cf-PWV, brachial-ankle PWV, femoral-tibial PWV, ankle-brachial index, pulse wave analysis (augmentation index and central pulse pressure) and aortic distensibility. ${ }^{24}$ As indicators of structural change in vascular stiffness, measurements of carotid artery intima-media thickness and vascular calcification scores will also be documented. ${ }^{26}$

\section{Healthy controls}

As controls, we will include adult individuals ( $\geq 18$ years of age) with measurements of validated indices of arterial stiffness who participated as healthy comparative controls in kidney donor studies. ${ }^{78}$

\section{Intervention}

In this review, uneventful unilateral nephrectomy for kidney donation independent of the surgical approach (laparoscopic and open nephrectomy) will be considered as the main intervention.

\section{Outcomes}

The primary outcome will be the difference in the functional metrics of arterial stiffness between healthy nephrectomised and non-nephrectomised participants as well as the difference in donors before and after nephrectomy. The absolute values in addition to between-group mean differences will be documented in their respective units of measurement. Secondary outcomes will be the differences in SBP and DBP $(\mathrm{mm} \mathrm{Hg})$, pulse pressure $(\mathrm{mm} \mathrm{Hg})$, serum creatinine levels $(\mu \mathrm{mol} / \mathrm{L})$, eGFR $\left(\mathrm{ml} / \mathrm{min} / 1.73 \mathrm{~m}^{2}\right)$, carotid artery intima-media thickness $(\mathrm{mm})$ and vascular calcification (scores). ${ }^{16} 242627$ Clinical outcomes and any additional measures of endothelial function will be documented and reported accordingly. ${ }^{1728}$

\section{cf-PWV reference group}

In addition to the primary comparative analysis, we will search for studies that measured cf-PWV in different healthy populations worldwide and who have reported values of normal cf-PWV standardised by age.${ }^{29} \mathrm{We}$ expect 
Table 1 List of inclusion and exclusion criteria for the selection of comparative groups

\begin{tabular}{|c|c|c|}
\hline & Inclusion criteria & Exclusion criteria \\
\hline Population & $\begin{array}{l}\text { Healthy subjects ( } \geq 18 \text { years) who underwent unilateral } \\
\text { simple nephrectomy as selected candidates that met } \\
\text { standard criteria for kidney donation. }\end{array}$ & $\begin{array}{l}\text { Healthy participants that underwent unilateral } \\
\text { simple nephrectomy other than for kidney } \\
\text { donation. } \\
\text { Children and adolescents with solitary kidney } \\
\text { after unilateral nephrectomy. }\end{array}$ \\
\hline Intervention & - Open or laparoscopic unilateral simple nephrectomy. & \\
\hline Outcome & $\begin{array}{l}\text { Functional indices of arterial stiffness: carotid-femoral } \\
\text { PWV, carotid-radial PWV, brachial-ankle PWV, femoral- } \\
\text { tibial PWV, pulse wave analysis (augmentation index } \\
\text { and central pulse pressure), ankle-brachial index, aortic } \\
\text { distensibility. } \\
\text { Structural indices of arterial stiffness: carotid artery } \\
\text { intima-media thickness and arterial calcification scores. }\end{array}$ & - None \\
\hline $\begin{array}{l}\text { Study } \\
\text { design }\end{array}$ & $\begin{array}{l}\text { Prospective non-randomised (cohort, case-control, case } \\
\text { series and before-and-after studies) and retrospective } \\
\text { studies if } 10 \text { or more participants have been included in } \\
\text { the primary analysis. } \\
\text { Articles reported in English, French, Italian, Portuguese } \\
\text { and Spanish languages. }\end{array}$ & $\begin{array}{l}\text { Paediatric and non-human studies } \\
\text { Narrative reviews } \\
\text { In vitro or mathematical modelling reports. } \\
\text { Duplicates } \\
\text { - Substudies of previously published trials. } \\
\text { Abstracts, conference proceedings. }\end{array}$ \\
\hline
\end{tabular}

PWW, pulse wave velocity.

that this analysis will improve the generalisability of this review.

\section{Study design}

Since prospective randomised clinical trials of donation would never be possible for ethical reasons, only prospective non-randomised (cohort, case-control, case series and before-and-after studies) and retrospective studies will be included, provided that 10 or more subjects have participated in the primary analysis.

\section{Search strategy}

Our search strategy will be conducted using MEDLINE, EMBASE, Cochrane Central databases, CADTH's 'Grey matters light', OVID, EBM reviews, Health Technology Assessment database and other grey literature of studies including government reports and policy statements published after 1 January 1960. A comprehensive search strategy will be constructed and implemented by a health information specialist (RS) with systematic review experience, in collaboration with the research team. MeSH terms will be used to capture the principal elements of the research question and identified citations will be exported to an excel spreadsheet for study review and selection. Our proposed literature search strategy is outlined in online supplemental appendix 2. Our planned systematic search will proceed until 15 March 2021. Manual review of the reference lists for all studies will be conducted according to predefined screening criteria (table 1).
A final 'grey literature' search will be conducted using 'Google Scholar' as well as reviews of ClinicalTrials.gov for any current study. No language restriction will be used in any of the initial search, but our final analysis will be restricted to articles reported in English, French, Italian, Portuguese and Spanish languages. Duplicate citations will be removed and search strategies will be kept up to the time of the end of this review.

\section{Study screening, exclusions and selection}

During the screening phase, we will include all prospective and retrospective non-randomised studies reporting measurements of arterial stiffness in nephrectomised and non-nephrectomised healthy participants. An iterative process of study selection will be followed using the inclusion and exclusion criteria set out in table 1 . We will exclude studies reporting on investigations in paediatric populations (age $<18$ years), non-human studies, narrative reviews, in vitro or mathematical modelling reports, abstracts or conference proceedings and any duplicate or substudy of previously published investigations. All review processes will be independently performed by two individuals, with a third person available for consensus in cases of discrepancies.

All citations will first be screened by title and abstract. After selection by title/abstract and with the purpose of facilitating a final decision on eligible studies, an intermediate screening of the material and methods section 
will be implemented in duplicate to confirm the type of methodology employed for measuring arterial stiffness. A judicious decisive selection before data extraction will be achieved by the independent reviewers at the full-text level. In cases of missing information, we will consider contacting study authors. Neither of the review authors will be blinded to the journal titles or to the study authors or institutions.

\section{Data extraction}

A data extraction form will be prepared a priori with consensus from all investigators and optimised using a subset of 4 randomly selected studies prior to duplicate extraction by two independent reviewers. When multiple publications arise from one study, relevant data will be extracted into a single form. Data extraction will include: (1) study characteristics, design and methods: title, authors, journal/source/year, language of publication, country, type of study design, study period, publication status, total number of donors and non-donors, inclusion and exclusion criteria, and points of measurement; (2) sample characteristics: age, sex, age at time of nephrectomy, age at time of assessment, duration of follow-up and type of arterial stiffness instrumentation; (3) outcomes: change in arterial stiffness based on the metric of measurement: cf-PWV, brachial-ankle PWV, femoral-tibial PWV, ankle-brachial index, augmentation index, central pulse pressure, aortic distensibility, carotid artery intimamedia thickness; arterial calcification scores, serum creatinine levels, eGFR, SBP, DBP and pulse blood pressure. We will document if arterial stiffness values are reported as adjusted or non-adjusted according to changes in mean arterial blood pressure and/or heart rate. If reported, we will document type and number of medications for the control of blood pressure.

\section{Risk assessment of bias}

Risk of bias for non-randomised studies will be assessed using the Risk of Bias in Non-Randomised studies (ROBINS-I) tool. ${ }^{30}$ Seven domains through which bias might be introduced will be independently evaluated and discussed by two reviewers, encompassing confounding, selection of participants (nephrectomised and nonnephrectomised), classification of the intervention (ie, unilateral nephrectomy), deviation from the intended intervention, missing data, measurement of outcomes and selection of the reported results. Each domain will be judged as either low, moderate, serious or critical risk of bias or no information available. After discussion among reviewers, an overall assessment of study bias with inclusion of all domains will be tabulated. Unresolved disagreements will be resolved with participation of a third reviewer.

\section{Data synthesis and analysis plan}

Studies will be included in qualitative and quantitative syntheses if they fulfil all eligibility criteria. Study characteristics will be summarised using means and SD or median and interquartile ranges for continuous variables and numbers and percentages for categorical variables. A narrative report of study characteristics will also be provided. We will identify potential sources of clinical heterogeneity according to differences in study design characteristics, methodological quality, characteristics at baseline between nephrectomised groups and their controls, and duration of follow-up periods. If at least two studies report on the same outcome, a quantitative analysis (ie, meta-analysis) will be attempted on those studies. Statistical heterogeneity will be characterised with Forest plots, the $\mathrm{I}^{2}$ and Cochran's $\mathrm{Q}$ statistics $(\mathrm{p}<0.10)$. If both clinical and statistical heterogeneity are acceptable, inverse variance-weighted effects meta-analysis will be performed. An $\mathrm{I}^{2}$ value less than $60 \%$ with a non-significant $\chi^{2}$ statistic $(p>0.10)$ will be suggestive of moderate statistical heterogeneity. We will primarily choose the random effects model according to the methodology of DerSimonian and Laird, ${ }^{31}$ but a fixed-effects meta-analysis will also be modelled as part of our sensitivity analysis. Based on the scale of measurements, we may choose to calculate pooled effect estimates using either standardised or mean differences and their corresponding 95\% confidence intervals. To minimise the 'double counting' error in before-and-after studies, half of the total number of study participants will be allocated to each study arm. All analysis will be performed using RevMan V.5.3 (The Nordic Cochrane Centre, The Cochrane Collaboration, 2014, Copenhagen).

\section{Sensitivity analysis}

Depending on the number of eligible studies, sensitivity analyses may be performed to evaluate the effects of extreme values, high risk of study bias, and any confounding effects associated with differences in the reporting of adjusted or non-adjusted values of arterial stiffness according to changes in mean arterial pressure and/or heart rate. An analysis of the differences between fixed and random effects models on the pooled effect estimates will also be assessed.

\section{Subgroup analysis}

Clinical studies ${ }^{32}$ have documented that living donors genetically related to their ESRD recipients are more likely to develop the primary kidney disease. Thus, it is critical to differentiate the effects of unilateral nephrectomy on arterial stiffness between recipient-related healthy controls and those who are not related to the recipient. To minimise bias in our review and depending on the number of eligible studies, nature and detail of the reported information, we will attempt to determine the effects of potential confounders by performing subgroup analyses based on age, surgical technique (open vs laparoscopic nephrectomy), duration of follow-up after nephrectomy (short vs long-term), type of comparator (recipient related vs non-recipient -related), number and type of medications used for the control of blood pressure and sex according to different male to female 
ratios. Intergroup differences will be analysed using the Cochran's Q statistics with $\mathrm{p} \leq 0.10$.

\section{Confidence in cumulative evidence}

To assess the certainty in the evidence and strength of recommendations on the effects of unilateral nephrectomy in kidney donors, two reviewers will evaluate quality of evidence for each outcome measure according to the five domains of Grading of Recommendations Assessment, Development and Evaluation recommendations. ${ }^{33}$

\section{Amendments}

Any protocol amendments will be summarised in the form of a table, where date of amendment, description of changes and rationale will be provided.

\section{Patient and public involvement}

Patients and/or the public were not involved in the design of the protocol.

\section{DISCUSSION}

Because living kidney donors are selected among the healthiest individuals, the long-term risk of ESRD, cardiovascular disease or death has been reported to be similar to or even lower than that in the general population. ${ }^{6}$ It has been documented, however, that unilateral nephrectomy frequently leads to reduced kidney function and variable levels of increased serum creatinine. ${ }^{51516}$ Although the mediumterm and long-term adverse effects of hyperfiltration and compensatory hypertrophy after donation remain unclear, endothelial dysfunction associated with decreased brachial artery reactivity ${ }^{1734}$ and increased levels of uremic toxins ${ }^{35}$ have been reported in kidney donors. More recently, some studies have reported that unilateral nephrectomy leads to an increase in aortic stiffness, left ventricle mass and decreased GFR as early as 1 year after surgery. ${ }^{7-9}$ Although blood pressure was not markedly affected in these studies, initial results suggest that reduced GFR may be an independent graded risk factor for increased aortic stiffness and adverse left ventricle remodelling, independent of blood pressure. $^{81112}$

To characterise the haemodynamic effects of unilateral nephrectomy in kidney donors, we propose to systematically identify, appraise and summarise all non-randomised studies that have investigated indices of arterial stiffness before and after nephrectomy in donors to determine any increase in the progression of arterial stiffness compared with age-matched healthy individuals. In addition to improving our understanding of the relationship between reduced kidney function after donation and cardiovascular function, our findings will determine if unilateral nephrectomy is a risk factor for increased arterial stiffness in donors and whether non-invasive metrics of arterial stiffness could be useful for risk stratification.

Animal studies have shown that alterations in renal development resulting in reduced nephron number in the offspring result in blood pressure elevation, reduced cardiac functional reserve and left ventricle hypertrophy in adulthood. ${ }^{36}$ Although alterations of the renin-angiotensin-aldosterone system and changes in vascular tone are frequently present after unilateral nephrectomy, ${ }^{37}$ kidney donation is not considered a risk factor for hypertension post-donation. Kasiske $e t a l^{38}$ followed living kidney donors for a 3-year period and identified an increase in blood pressure over time, but differences between donors and controls were non-significant. Kim $e t a \hat{l}^{39}$ found that there was an increase in blood pressure after donation with $21 \%$ of donors requiring medication for control of hypertension. Thiel $e t a l^{13}$ reported that the risk of developing hypertension (defined as $>140 / 90 \mathrm{~mm} \mathrm{Hg}$ ) increased 3.6-fold 1-year postdonation, but baseline values of kidney donors who became hypertensive were significantly higher than those who were less susceptible to hypertension. Because systemic blood pressure may be a determinant of arterial stiffness, we will include SBP and DBP as a secondary outcome. This will add to the understanding of the potential relationship between blood pressure and arterial stiffness following organ donation. ${ }^{37-39}$

To accept that increased arterial stiffness is associated with the effects of unilateral nephrectomy, convincing evidence based on good-quality studies and adequate methodological designs is critical. Although quality of evidence and differences in study design are factors that may limit the strength of our associations, implementing well-validated scales in our study for the assessment of risk of bias and certainty of evidence will minimise misinterpretations. ${ }^{30} 33$ In cases where information is not available, we will make every possible effort to contact the primary author(s) to obtain any missing information.

Living kidney donors obtain no medical benefit from donation, exposing themselves to health risks associated with the surgical procedure and the effects of reduced renal mass for the benefit of another human being. Promotion of donor autonomy in the medical decision-making process requires accurate quantification of the mid and long-term risks after donation. This is particularly critical for young living donors who have the longest risk of exposure. ${ }^{39} 40$ Our review will provide a more accurate estimate of the 'short-to-mid-term' risks of cardiovascular disease after donation by examining the progression of arterial stiffness postnephrectomy. ${ }^{37-41}$ Such results can inform potential donors and may guide their follow-up care and preserve good health in the long -term.

\section{Ethics and dissemination}

No ethics approval is required for this systematic review. Our findings will be disseminated through peer-review publication and presentation, which can inform clinical guidelines and management of potential kidney donors.

Contributors RAR and KDB conceived and designed the study; RAR created the analytical plan and drafted the protocol; RAR and RS designed the search strategy; RAR, MTS, MA and KDB will be involved in one or more of the following tasks: study screening and selection, data extraction, verification, quality appraisal, synthesis, analysis of the evidence and data interpretation. All authors have read, reviewed and approved the final version of the manuscript. RAR is the guarantor. 
Funding This work is supported by Kidney Research Centre, Ottawa Hospital Research Institute, University of Ottawa.

Competing interests None declared.

Patient consent for publication Not required.

Provenance and peer review Not commissioned; externally peer reviewed.

Supplemental material This content has been supplied by the author(s). It has not been vetted by BMJ Publishing Group Limited (BMJ) and may not have been peer-reviewed. Any opinions or recommendations discussed are solely those of the author(s) and are not endorsed by BMJ. BMJ disclaims all liability and responsibility arising from any reliance placed on the content. Where the content includes any translated material, BMJ does not warrant the accuracy and reliability of the translations (including but not limited to local regulations, clinical guidelines, terminology, drug names and drug dosages), and is not responsible for any error and/or omissions arising from translation and adaptation or otherwise.

Open access This is an open access article distributed in accordance with the Creative Commons Attribution Non Commercial (CC BY-NC 4.0) license, which permits others to distribute, remix, adapt, build upon this work non-commercially, and license their derivative works on different terms, provided the original work is properly cited, appropriate credit is given, any changes made indicated, and the use is non-commercial. See: http://creativecommons.org/licenses/by-nc/4.0/.

\section{ORCID iD}

Rosendo A Rodriguez http://orcid.org/0000-0002-6039-9274

\section{REFERENCES}

1 Foroutan F, Friesen EL, Clark KE, et al. Risk factors for 1-year graft loss after kidney transplantation: systematic review and metaanalysis. Clin J Am Soc Nephrol 2019;14:1642-50.

2 Keys DO, Jackson S, Berglund D, et al. Kidney donor outcomes $\geq 50$ years after donation. Clin Transplant 2019;33:e13657.

3 Matas AJ, Smith JM, Skeans MA, et al. OPTN/SRTR 2012 annual data report: kidney. Am J Transplant 2014;14 Suppl 1:11-44.

4 Horvat LD, Shariff SZ, Garg AX. Donor nephrectomy outcomes research: global trends in the rates of living kidney donation. Kidney Int 2009; 75:1088-98.

5 Maggiore U, Budde K, Heemann U, et al. Long-Term risks of kidney living donation: review and position paper by the ERA-EDTA Descartes Working group. Nephrol Dial Transplant 2017;32:216-23.

6 O'Keeffe LM, Ramond A, Oliver-Williams C, et al. Mid- and long-term health risks in living kidney donors: a systematic review and metaanalysis. Ann Intern Med 2018;168:276-84.

7 Bahous SA, Stephan A, Blacher J, et al. Aortic stiffness, living donors, and renal transplantation. Hypertension 2006;47:216-21.

8 Moody WE, Ferro CJ, Edwards NC, et al. Cardiovascular effects of unilateral nephrectomy in living kidney donors. Hypertension 2016;67:368-77.

9 Buus NH, Carlsen RK, Hughes AD, et al. Influence of renal transplantation and living kidney donation on large artery stiffness and peripheral vascular resistance. Am J Hypertens 2020;33:234-42.

10 Ohyama Y, Ambale-Venkatesh B, Noda C, et al. Association of aortic stiffness with left ventricular remodeling and reduced left ventricular function measured by magnetic resonance imaging: the multi-ethnic study of atherosclerosis. Circ Cardiovasc Imaging 2016;9.

11 Gokalp C, Guner Oytun M, Gunay E, et al. Increase in interventricular septum thickness may be the first sign of cardiovascular change in kidney donors. Echocardiography 2020;37:276-82.

12 Williams SL, Oler J, Jorkasky DK. Long-Term renal function in kidney donors: a comparison of donors and their siblings. Ann Intern Med 1986;105:1-8.

13 Thiel GT, Nolte C, Tsinalis D, et al. Investigating kidney donation as a risk factor for hypertension and microalbuminuria: findings from the Swiss prospective follow-up of living kidney donors. BMJ Open 2016;6:e010869.

14 Vlachopoulos C, Aznaouridis K, Stefanadis C. Prediction of cardiovascular events and all-cause mortality with arterial stiffness: a systematic review and meta-analysis. J Am Coll Cardio 2010;55:1318-27.

15 Chronic Kidney Disease Prognosis Consortium, Matsushita K, van der Velde $\mathrm{M}$, et al. Association of estimated glomerular filtration rate and albuminuria with all-cause and cardiovascular mortality in general population cohorts: a collaborative meta-analysis. Lancet 2010;375:2073-81.

16 Garg AX, Meirambayeva A, Huang A, et al. Cardiovascular disease in kidney donors: matched cohort study. BMJ 2012;344:e1203.
17 Yilmaz BA, Caliskan Y, Yilmaz A, et al. Cardiovascular-renal changes after kidney donation: one-year follow-up study. Transplantation 2015;99:760-4

18 Sidibé A, Fortier C, Desjardins Marie-Pier, Desjardins MP, et al. Reduction of arterial stiffness after kidney transplantation: a systematic review and Meta-Analysis. J Am Heart Assoc 2017;6:007235.

19 Rodriguez RA, Hae R, Spence M, et al. A Systematic Review and Meta-analysis of Nonpharmacologic-based Interventions for Aortic Stiffness in End-Stage Renal Disease. Kidney Int Rep 2019;4:1109-21.

20 Reeves B, Deeks JJ, Higgins J. Including non-randomized studies on intervention effects. In: Higgins J, Thomas J, eds. Cochrane Handbook for systematic reviews of interventions. Version 6.0. Hoboken: John Wiley \& Sons, 2019. https://training.cochrane.org/ handbook/current

21 National Institute for Health Research. Prospero International prospective register of systematic reviews, 2020. Available: https:// www.crd.york.ac.uk/prospero/

22 Moher D, Liberati A, Tetzlaff J, et al. Preferred reporting items for systematic reviews and meta-analyses: the PRISMA statement. $J$ Clin Epidemiol 2009;62:1006-12.

23 Beller EM, Glasziou PP, Altman DG, et al. PRISMA for Abstracts: reporting systematic reviews in Journal and conference Abstracts. PLoS Med 2013;10:e10001419.

24 DeLoach SS, Townsend RR. Vascular stiffness: its measurement and significance for epidemiologic and outcome studies. Clin J Am Soc Nephrol 2008;3:184-92.

25 Redheuil A, Wu CO, Kachenoura N, et al. Proximal aortic distensibility is an independent predictor of all-cause mortality and incident cv events: the MESA study. J Am Coll Cardiol 2014;64:2619-29.

26 Polak JF, O'Leary DH. Carotid intima-media thickness as surrogate for and predictor of CVD. Glob Heart 2016;11:295-312.

27 Levey AS, Stevens LA. Estimating GFR using the CKD epidemiology collaboration (CKD-EPI) creatinine equation: more accurate GFR estimates, lower CKD prevalence estimates, and better risk predictions. Am J Kidney Dis 2010;55:622-7.

28 Celermajer DS, Sorensen KE, Gooch VM, et al. Non-Invasive detection of endothelial dysfunction in children and adults at risk of atherosclerosis. Lancet 1992;340:1111-5.

29 Reference Values for Arterial Stiffness' Collaboration. Determinants of pulse wave velocity in healthy people and in the presence of cardiovascular risk factors: 'establishing normal and reference values'. Eur Heart J 2010;31:2338-50.

30 Sterne JA, Hernán MA, Reeves BC, et al. ROBINS-I: a tool for assessing risk of bias in non-randomised studies of interventions. BMJ 2016;355:i4919.

31 DerSimonian R, Laird N. Meta-Analysis in clinical trials revisited. Contemp Clin Trials 2015;45:139-45.

32 Skrunes R, Svarstad E, Reisæter AV, et al. Familial clustering of ESRD in the Norwegian population. Clin J Am Soc Nephrol 2014;9:1692-700.

33 Guyatt GH, Oxman AD, Vist GE, et al. Grade: an emerging consensus on rating quality of evidence and strength of recommendations. $B M J$ 2008;336:924-6.

34 Söğütdelen E, Yildirim T, Haberal HB, et al. Donor nephrectomy may compromise the cardiovascular system: a retrospective, singlecenter study. Exp Clin Transplant 2018. doi:10.6002/ect.2018.0060. [Epub ahead of print: 06 Aug 2018].

35 Rossi M, Campbell KL, Johnson DW, et al. Uremic toxin development in living kidney donors: a longitudinal study. Transplantation 2014;97:548-54.

36 Singh RR, Jefferies AJ, Lankadeva YR, et al. Increased cardiovascular and renal risk is associated with low nephron Endowment in aged females: an ovine model of fetal unilateral nephrectomy. PLoS One 2012;7:e42400.

37 Rastogi A, Yuan S, Arman F, et al. Blood pressure and living kidney donors: a clinical perspective. Transplant Direct 2019;5:e488.

38 Kasiske BL, Anderson-Haag T, Israni AK, et al. A prospective controlled study of living kidney donors: three-year follow-up. Am J Kidney Dis 2015;66:114-24.

$39 \mathrm{Kim} \mathrm{SH}$, Hwang HS, Yoon HE, et al. Long-Term risk of hypertension and chronic kidney disease in living kidney donors. Transplant Proc 2012;44:632-4.

40 Locke JE, Sawinski D, Reed RD, et al. Apolipoprotein L1 and chronic kidney disease risk in young potential living kidney donors. Ann Surg 2018;267:1161-8.

41 Najarian JS, Chavers BM, McHugh LE, et al. 20 years or more of follow-up of living kidney donors. Lancet 1992;340:807-10. 